\title{
DECISION-MAKING OF ACCUSED PERSONS WITH PERSONALITY DISORDERS
}

Gorinov V.V., Shekhovtsova E.S.

Objective. Refinement of the possibilities of using psychological diagnostic methods of personality traits and regulation of behavior.

Background. There are a lot of quite difference options of dysregulation in personality disorder and they are associated with the level of personal disharmony and the degree of complexity of the situation of choice. Personality disorders are includes the lack of stable semantic formations, prognostic abilities, emotional experience, motivational simplicity, the discrepancy between the ideal goal and the real ways of achieving it.

Materials and methods. Clinical and psychopathological method, psychological method

\section{Results}

Accused persons with personality disorders tend to be impulsive; they have difficulties of emotional control, immediate stress response, a lack of rational analysis of situational conditions, the prevalence of unconscious response models in conflict situations. The basic beliefs of the accused with personality disorders include instability of self-esteem, rigidity of assessments. So it was found that these features are associated with an increased risk of committing rash acts, including aggressive and criminal nature.

In view of the type of crime committed, the following were found. Persons who have committed crimes against sexual integrity are more flexible in matters of interpersonal relations than groups of persons who have committed murder and serious harm to health, and crimes related to the illegal acquisition, storage, transportation, manufacture, processing of drugs.

\section{Conclusions}

Thus, it was found that psychological methods for diagnosing decision-making features and personal characteristics are a promising direction in creating a comprehensive diagnostic system for forensic psychiatric assessment of personality disorders. 